\title{
Blood Plasma from Survivors of COVID-19: A Novel and Next Frontier Approach to Fight against Pandemic Coronavirus
}

\author{
Nimesh Singh ${ }^{1^{*}}$ and Archna Pandey ${ }^{2}$ \\ ${ }^{1}$ Flax Laboratories, Mahad MIDC, Maharashtra, India \\ ${ }^{2}$ Department of Chemistry, Dr. Harisingh Gour University, Madhya Pradesh, India \\ *Corresponding author: Dr. Nimesh Singh, Flax Laboratories, Mahad MIDC, Taluka-Mahad, Distt - Raigad, Maharashtra, \\ India
}

\begin{abstract}
Blood from people who have recovered can be a rich source of antibodies, proteins made by the immune system to attack the virus. The part of the blood that contains antibodies, so-called convalescent plasma, has been used for decades to treat infectious diseases, including Ebola and influenza. In the absence of a cure or vaccine for the coronavirus, a group of scientists is searching for a fast solution in an unconventional place: The veins of people who have recovered. Starting in New York City hospitals this week, and soon in dozens of medical centers across the United States, researchers will start drawing blood from COVID-19 survivors, who have antibodies their bodies made to fight the disease. They'll then isolate their plasma, the liquid part of blood that contains antibodies. And in a process called "convalescent plasma therapy," their antibodies will be transferred to others, either to protect them against getting infected or to boost the immune systems of those who are already sick. This highly experimental therapy hasn't been proven to work against the coronavirus, but preliminary research out of China suggests that it has helped a small group of patients recover. It's also seen some success in past infectious disease outbreaks, including in fighting the coronavirus that caused the SARS outbreak.
\end{abstract}

\section{Keywords}

Antibody, Antigen, Plasma, RBCs, SARS-CoV, Chromatography, Precipitation

\section{Introduction}

The novel coronavirus 2019-nCoV has recently emerged as a human pathogen in the city of Wuhan in China's Hubei province, causing fever, severe respiratory illness, and pneumonia - a disease recently named
COVID-19 [1,2]. According to the World Health Organization (WHO), as of 16 February 2020, there had been $>51,000$ confirmed cases globally, leading to at least 1600 deaths. The emerging pathogen was rapidly characterized as a new member of the betacoronavirus genus, closely related to several bat coronaviruses and to severe acute respiratory syndrome coronavirus (SARS-CoV) $[3,4]$. Compared with SARSCoV, 2019-nCoV appears to be more readily transmitted from human to human, spreading to multiple continents and leading to the WHO's declaration of a public health emergency of international concern (PHEIC) on 30 January $2020[1,5,6]$.

Since 2013, porcine epidemic diarrhea coronavirus (PEDV) has swept throughout the United States, causing an almost $100 \%$ fatality rate in piglets and wiping out more than $10 \%$ of America's pig population in less than a year [7-9]. In general, coronaviruses cause widespread respiratory, gastrointestinal, and central nervous system diseases in humans and other animals, threatening human health and causing economic loss $[10,11]$. Coronaviruses are capable of adapting to new environments through mutation and recombination with relative ease and hence are programmed to alter host range and tissue tropism efficiently [12-14]. Therefore, health threats from coronaviruses are constant and long-term. Understanding the virology of coronaviruses and controlling their spread have important implications for global health and economic stability.

Citation: Singh N, Pandey A (2020) Blood Plasma from Survivors of COVID-19: A Novel and Next Frontier Approach to Fight against Pandemic Coronavirus. Int J Immunol Immunother 7:045. doi. org/10.23937/2378-3672/1410045

Received Date: April 04, 2020: Accepted: April 24, 2020: Published: April 26, 2020

Copyright: (c) 2020 Singh N. This is an open-access article distributed under the terms of the Creative Commons Attribution License, which permits unrestricted use, distribution, and reproduction in any medium, provided the original author and source are credited. 
Coronaviruses are large, enveloped, positive-stranded RNA viruses. They have the largest genome among all RNA viruses, typically ranging from 27 to $32 \mathrm{~kb}$. The genome is packed inside a helical capsid formed by the nucleocapsid protein $(\mathrm{N})$ and further surrounded by an envelope. Associated with the viral envelope are at least three structural proteins: The membrane protein (M) and the envelope protein (E) are involved in virus assembly, whereas the spike protein (S) mediates virus entry into host cells. Some coronaviruses also encode an envelope-associated hemagglutinin-esterase protein (HE). Among these structural proteins, the spike forms large protrusions from the virus surface, giving coronaviruses the appearance of having crowns (hence their name; corona in Latin means crown). In addition to mediating virus entry, the spike is a critical determinant of viral host range and tissue tropism and a major inducer of host immune responses. The coronavirus spike contains three segments: A large ectodomain, a single-pass transmembrane anchor, and a short intracellular tail. The ectodomain consists of a receptor-binding subunit S1 and a membrane-fusion subunit S2. Electron microscopy studies revealed that the spike is a clove-shaped trimer with three S1 heads and a trimeric S2 stalk [1518]. During virus entry, S1 binds to a receptor on the host cell surface for viral attachment, and S2 fuses the host and viral membranes, allowing viral genomes to enter host cells. Receptor binding and membrane fusion are the initial and critical steps in the coronavirus infection cycle; they also serve as primary targets for human inventions. In this article, I review the structure and function of coronavirus spikes and discuss their evolution.

An old idea for fighting infections - an approach most physicians know about only from medical lore - is being revived as people wait for drugs and vaccines to thwart the novel coronavirus. If it works, the blood plasma of people who have recovered from covid-19 would be used to protect health-care workers and help sick people get well. The possible therapy is based on a medical concept called "passive immunity". People who recover from an infection develop antibodies that circulate in the blood and can neutralize the pathogen. Infusions of plasma - the clear liquid that remains when blood cells are removed - may increase people's disease-fighting response to the virus, giving their immune systems an important boost. The approach has been used against polio, measles, mumps and flu. Plasma could be used to treat people who are sick and to prevent illness in health-care workers, Casadevall said, especially those at greatest risk for developing the illness because of repeated exposure.

First, experts must develop tests to measure the levels of antibodies, and then use those to identify donors whose plasma is rich in antibodies that could help others battle the illness. Then, they have to deliver the plasma to patients - most likely in clinical trials designed to measure whether it works. The plasma must be safe and disease-free, not only from other blood-borne pathogens but also from the novel coronavirus.

Convalescent plasma has a real role - this has been going on for over 100 years. We know this stuff works," said Wayne A. Marasco, an infectious disease physician at Dana-Farber Cancer Institute in Boston. "If you do this right and harvest plasma from someone who has undergone infection, you can get protective antibodies that can be infused in other people".

\section{What is Plasma?}

Blood fractionation is the process of fractionating whole blood, or separating it into its component parts. This is typically done by centrifuging the blood.

The resulting components are:

- A clear solution of blood plasma in the upper phase (which can be separated into its own fractions, see Blood plasma fractionation),

- The buffy coat, which is a thin layer of leukocytes (white blood cells) mixed with platelets in the middle, and

- Erythrocytes (red blood cells) at the bottom of the centrifuge tube.

\section{Separation and Purification of Antibodies from Blood Plasma}

Plasma proteins are separated by using the inherent differences of each protein. Fractionation involves changing the conditions of the pooled plasma (e.g., the temperature or the acidity) so that proteins that are normally dissolved in the plasma fluid become insoluble, forming large clumps, called precipitate. The insoluble protein can be collected by centrifugation. One of the very effective ways for carrying out this process is the addition of alcohol to the plasma membrane pool while simultaneously cooling the pool. This process is sometimes called cold alcohol fractionation or ethanol fractionation. It was described by and bears the eponym of Dr. Edwin J. Cohn. This procedure is carried out in a series of steps so that a single pool of plasma yields several different protein products, such as albumin and immune globulin [1,2]. Human serum albumin prepared by this process is used in some vaccines, for treating burn victims, and other medical applications.

Antibody detection and identification are processes that are commonly performed in the transfusion service before transfusion of allogeneic red blood cells (RBCs). Antibody identification usually follows the discovery of a positive antibody detection test, or other factors such as $A B O$ serum/cell discrepancy or an incompatible crossmatch. Antibody identification is a necessary practice in blood banking to determine the suitability of blood products for transfusion on an individual basis. When the presence of multiple antibodies is suspected, seve- 
ral methods, including neutralization of patient's plasma, titration, elution, chemical or enzyme treatment of reagent RBCs, and adsorption with allogeneic RBCs, may be used to separate and properly identify other atypical antibodies that are present in a single serum or plasma sample. This review will focus on the use of allogeneic adsorption to identify antibody specificities in a patient's sample.

\section{Procedural steps}

- Select test RBCs to be used for allogeneic adsorption.

- Wash the test RBCs three to four times with phosphate-buffered saline.

- Remove all supernatant saline from the washed RBCs.

- If enzyme treatment is desired, follow manufacturer's directions and treat the RBCs.

- Wash the RBCs three to four times after treatment with enzymes.

- Add 1 volume serum or plasma to 1 volume washed, packed RBCs.

- Stopper, gently mix well, and incubate for 30-60 minutes at $37^{\circ} \mathrm{C}$.

- Centrifuge for 5 minutes and remove the plasma/serum. Test against fresh sample of adsorbing cells to see if adsorption is complete.

- Repeat the procedure with fresh adsorbing cells if adsorption is incomplete.

\section{Physicochemical fractionation antibody purifica- tion}

- Size exclusion chromatography.

- Ammonium sulfate precipitation.

- Ion exchange chromatography.

- Immobilized metal chelate chromatography.

- Thiophilic adsorption.

- Melon Gel chromatography.

- Protein $A, G$ and $L$ antibody-binding ligands.

- Antibody purification with Protein A, G and L.

Size exclusion chromatography: Dialysis, desalting and diafiltration can be used to exchange antibodies into particular buffers and remove undesired low-molecular weight (MW) components. Dialysis membranes, size-exclusion resins, and diafiltration devices that feature high-molecular weight cut-offs (MWCO) can be used to separate immunoglobulins (>140 kDa) from small proteins and peptides. However, except with specialized columns and equipment, these techniques alone cannot purify antibodies from other proteins and macromolecules that are present in typical antibody samples. More commonly, gel filtration and dialysis are used following other purification steps, such as ammonium sulfate precipitation [19].

Ammonium sulfate precipitation: Ammonium sulfate precipitation is frequently used to enrich and concentrate antibodies from serum, ascites fluid or cell culture supernatant. As the concentration of this lyotropic salt is increased in a sample, proteins and other macromolecules become progressively less soluble until they precipitate; the lyotropic effect is called "salting out". Antibodies precipitate at lower concentrations of ammonium sulfate than most other proteins and components of serum.

At 40 to $50 \%$ ammonium sulfate saturation (100\% saturation equals $4.32 \mathrm{M}$ ), immunoglobulins will precipitate while other proteins remain in solution [20]. The usual method involves very slowly adding an equal volume of saturated ammonium sulfate solution to a neutralized antibody sample, followed by incubation for several hours at room temperature or $4{ }^{\circ} \mathrm{C}$. After centrifugation and removal of the supernatant, the antibody-pellet is dissolved in buffer, such as phosphate-buffered saline (PBS).

The selectivity, yield, purity and reproducibility of precipitation depends upon several factors, including time, temperature, $\mathrm{pH}$ and rate of salt addition [21]. Ammonium sulfate precipitation provides sufficient purification for some antibody applications, but most often it is performed as a preliminary step before column chromatography or other purification method (e.g., Melon Gel Monoclonal IgG Purification Kit). Using partially-purified antibody samples can improve the performance and extend the life of affinity columns.

Other antibody precipitation reagents that have been used for special antibody purification situations include using octonoic acid, polyethylene glycol and ethacridine [21].

Ion exchange chromatography: Numerous chemically-based, solid-phase chromatography methods have been adapted and optimized to achieve antibody purification in particular situations.

Ion exchange chromatography (IEC) uses positively or negatively charged resins to bind proteins based on their net charges in a given buffer system $(\mathrm{pH})$. Especially in commercial operations involving production of monoclonal antibodies, conditions for IEC can be determined that bind and release the target antibody with a high degree of specificity. Conversely, conditions can be found that bind nearly all other sample components except antibodies. Once so optimized, IEC is a cost-effective, gentle and reliable method for antibody purification.

Immobilized metal chelate chromatography: Immobilized metal chelate chromatography (IMAC) uses chelate-immobilized divalent metal ions (usually nickel, 
$\mathrm{Ni}^{2+}$ ) to bind proteins or peptides that contain clusters of three or more consecutive histidine residues. The strategy is most often used to purify recombinant proteins that have been engineered to contain a terminal $6 \times$ His fusion tag. Interestingly, mammalian IgGs are one of the few abundant proteins in serum (or monoclonal hybridoma cell culture supernatant) that possess histidine clusters capable of being bound by immobilized nickel. As with IEC, IMAC conditions for binding and elution can be optimized for particular samples to provide gentle and reliable antibody purification [21]. For example, the Pierce Conjugate Purification Kit uses this technique to separate AP- or HRP-labeled (enzyme-conjugated) antibody from excess, non-conjugated enzyme following a labeling procedure.

Thiophilic adsorption: Thiophilic adsorption is a highly selective type of protein-ligand interaction that combines the properties of hydrophobic interaction chromatography (HIC) and ammonium sulfate precipitation (the lyotropic effect). The interaction is termed thiophilic because it involves the binding of proteins to a sulfone group in close proximity to a thioether. In contrast to strict HIC, thiophilic adsorption depends upon a high concentration of lyotropic salt (e.g., potassium sulfate as opposed to sodium chloride). With typical antibody samples that have been equilibrated with potassium sulfate, binding is quite specific to antibodies. After non-bound components are washed away, the antibodies are easily recovered with gentle elution conditions (e.g., $50 \mathrm{mM}$ sodium phosphate buffer, $\mathrm{pH} 7$ to 8). Thiophilic Adsorbent (also called T-Gel) is $6 \%$ beaded agarose modified to contain the sulfone-thioether ligand. The adsorbent has a high binding capacity and broad specificity toward imunoglobulins from various animal species. Notably, it is one of few affinity methods that is effective for chicken IgY purification.

Melon gel chromatography: Melon Gel is a proprietary resin chemistry (and optimized buffer system) for purifying antibodies by chemical-based fractionation. In the specified mild buffer condition, Melon Gel resin binds most non-IgG proteins found in serum, ascites fluid and culture supernatants, while allowing purified IgG to be collected in the flow-through fraction.

The various Melon Gel kits are optimized for rapid, convenient and gentle purification of IgG. The Melon Gel kit for purification of monoclonal antibodies illustrates the benefits of combining two purification techniques. Ammonium sulfate precipitation is recommended for cell culture supernatant samples before performing the final Melon Gel purification. Treatment of ascites fluid samples with a conditioning reagent is recommended before Melon Gel purification to decrease the co-purification of transferrin with the antibody.

Because the Melon Gel system uses negative selection and requires no elution steps, it also provides a convenient and effective method for removing bovine serum albumin (BSA) or gelatin from antibody stock solutions so that these stabilizing proteins will not interfere with antibody labeling procedures. This is the basis of our Antibody Clean-Up Kit.

If the specific removal of one particular undesirable serum component can be considered a form of antibody purification, then it is appropriate here to mention albumin removal. Albumin accounts for approx. $60 \%$ of human serum protein. Cibacron Blue Dye binds selectively to human serum albumin and can be used as an affinity ligand to prepare albumin-free serum samples for 2D electrophoretic analysis.

Protein A, G and L Antibody-binding ligands: Protein $A$, Protein $G$ and Protein $L$ are three bacterial proteins whose antibody-binding properties have been well characterized. These proteins have been produced recombinantly and used routinely for affinity purification of key antibody types from a variety of species. Most commercially-available, recombinant forms of these proteins have unnecessary sequences removed (including the HSA-binding domain from Protein G) and are therefore smaller than their native counterparts. A genetically-engineered recombinant form of Protein $A$ and Protein G, called Protein A/G, is also widely available. All four recombinant Ig-binding proteins are used routinely by researchers in numerous immune detection and immune affinity applications.

Antibody purification with Protein A, G and L: To accomplish antibody purification with Protein A, Protein G, Protein A/G or Protein L, they are covalently immobilized onto porous resins (such as beaded agarose) or magnetic beads. Because these proteins contain several antibody-binding domains, nearly every individual immobilized molecule, no matter its orientation maintains at least one functional and unhindered binding domain. Furthermore, because the proteins bind to antibodies at sites other than the antigen-binding domain, the immobilized forms of these proteins can be used in purification schemes, such as immuno precipitation, in which antibody binding protein is used to purify an antigen from a sample by binding an antibody while it is bound to its antigen.

Protein A, G, A/G and L have different binding properties, which make each one suitable for different types of antibody targets (e.g., antibody subclass or animal species). It is important to realize that use of Protein A, $G$ or $L$ results in purification of general immunoglobulin from a crude sample. Depending on the sample source, antigen-specific antibody may account for only a small portion of the total immunoglobulin in the sample. For example, generally only $2-5 \%$ of total IgG in mouse serum is specific for the antigen used to immunize the animal.

Using a column of Protein Aagarose resin and rabbit serum as the example, the general procedure for antibody purification with these ligands is as follows: 
1. Bind: Add a clarified, physiologic-buffered (pH 7 to 8) sample of rabbit serum to the column and allow it to slowly pass through or mix with the Protein A resin to allow the IgG to bind to the immobilized ligand.

2. Wash: Add phosphate-buffered saline (PBS) and allow it to pass through the column to wash away nonbound serum components. Use a volume of wash buffer equivalent to 5 to 10 times the resin volume.

3. Elute: Add acidic elution buffer (e.g., $0.1 \mathrm{M}$ glycine- $\mathrm{HCl}, \mathrm{pH} 2.8$ ), and collect small fractions of solution that pass from the column. The low-pH condition dissociates the antibody from the immobilized Protein $A$, and the IgG is recovered in its purified state in one or several of the collected fractions.

4. Neutralize or exchange buffer: Use a protein assay or other means to identify and combine elution fractions that contain the purified antibody. Then add $1 / 10^{\text {th }}$ volume of $1 \mathrm{M}$ Tris- $\mathrm{HCl}(\mathrm{pH} 8.5)$ to neutralize the buffer. Alternatively, use a desalting column or dialysis to exchange the purified antibody into a more suitable buffer for long-term storage.

\section{Purification of IgM}

Proteins $\mathrm{G}$ and $\mathrm{A}$ do not bind strongly with $\operatorname{Ig} \mathrm{M}$ as there is steric hindrance of the binding regions on IgM. Thus, to purification of IgM involves several methods, including ammonium sulfate precipitation, ion exchange chromatography, gel filtration, and zone electrophoresis.

\section{Purification of IgA}

This method was uncovered when a D-galactose-lectin called jacalin was extracted from jackfruit seeds. This compound was found to contain four identical domains, and it binds to IgA. Jacalin can be immobilized on agarose gels and subsequently can be used to purify and separate IgA from other immunoglobulins.

\section{Purification of IgY}

IgY is a unique immunoglobulin made by chickens, and it is present in high quantities in egg yolk. Proteins $A, G$, and $L$ cannot be used to purify IgY as these proteins do not bind to IgY. Thus, ammonium sulfate precipitation method is used to purify IgY.

\section{Antigen-Specific Affinity Purification of Anti- bodies}

As antibodies bind to a specific antigen, this interaction can also be used to purify antibodies. Affinity purification involves immobilizing an antigen which is specific for an antibody, such that only antibodies which bind to the specific antigen are isolated. This method is called ligand immobilization method for affinity purification.

\section{Monoclonal Antibodies to SARS-Associated Coronavirus (SARS-CoV)}

Monoclonal antibodies (Mabs) against the Urbani strain of the SARS-associated coronavirus (SARSCoV) were developed and characterized for reactivity to SARS-CoV and SARS-CoV S, N, M, and E proteins using enzyme-linked immunoabsorbent (ELISA), radioimmunoprecipitation, immunofluorescence, Western Blot and microneutralization assays. Twenty-six mAbs were reactive to SARS-CoV by ELISA, and nine were chosen for detailed characterization. Five mAbs reacted against the $S$ protein, two against the $M$ protein, and one each against the $\mathrm{N}$ and $\mathrm{E}$ proteins. Two of five $S$ protein mAbs neutralized SARS-CoV infection of Vero E6 cells and reacted to an epitope within amino acids $490-510$ in the $S$ protein. While two of the three non-neutralizing antibodies recognized at second epitope within amino acids 270-350. The mAbs characterized should prove useful for developing SARS-CoV diagnostic assays and for studying the biology of infection and pathogenesis of disease [22].

\section{Conclusion}

Antibodies are an important component in host immune responses to viral pathogens. Because of their unique maturation process, antibodies can evolve to be highly specific to viral antigens. Physicians and researchers have been relying on such high specificity in their quest to understand host-viral interaction and viral pathogenesis mechanisms and to find potential cures for viral infection and disease. The epidemiological impact of viral diseases, combined with the emergence and reemergence of some viruses, and the difficulties in identifying effective therapies, have encouraged several studies to develop new therapeutic strategies for viral infections. In this context, the use of immunotherapy for the treatment of viral diseases is increasing. One of the strategies of immunotherapy is the use of antibodies, particularly the monoclonal antibodies (mAbs) and multi-specific antibodies, which bind directly to the viral antigen and bring about activation of the immune system. In current review article we proposed use of antibodies from isolated from blood of person who recently survived from COVID-19 infection. Different types of method described for isolation and purification of antibodies for treatment of global pandemic Coronavirus.

\section{Acknowledgement}

This review article was supported by Flax Laboratories, B-29/1, Mahad MIDC, Taluka- Mahad, Distt. - Raigad, M.H., INDIA. Also author would like to acknowledge Prof. Archna Pandey from Department of Chemistry, Dr. H.S. Gour University, Sagar, M.P. INDIA for her kind support during review of article. 


\section{References}

1. Jasper Fuk-Woo Chan, Shuofeng Yuan, Kin-Hang Kok, Kelvin Kai-Wang, Hin Chu, et al. (2020) A familial cluster of pneumonia associated with the 2019 novel coronavirus indicating person-to-person transmission: A study of a family cluster. Lancet 395: 514-523.

2. Chaolin Huang, Yeming Wang, Xingwang Li, Lili Ren, Jianping Zhao, et al. (2020) Clinical features of patients infected with 2019 novel coronavirus in Wuhan, China. Lancet 395 : 497-506.

3. Roujian Lu, Xiang Zhao, Juan Li, Peihua Niu, Bo Yang, et al. (2020) Genomic characterisation and epidemiology of 2019 novel coronavirus: Implications for virus origins and receptor binding. Lancet 20: 30251-30258.

4. Wu F, Zhao S, Yu B, Chen YM, Wang W, et al. (2020) A new coronavirus associated with human respiratory disease in China. Nature.

5. Nanshan Chen, Min Zhou, Xuan Dong, Jieming Qu, Fengyun Gong, et al. (2020) Epidemiological and clinical characteristics of 99 cases of 2019 novel coronavirus pneumonia in Wuhan, China: A descriptive study. Lancet 395: 507-513.

6. Qun Li, Xuhua Guan, Peng Wu, Xiaoye Wang, Lei Zhou, et al. (2020) Early transmission dynamics in Wuhan, China, of Novel Coronavirus-infected pneumonia. N Engl J Med.

7. Mole B (2013) Deadly pig virus slips through US borders. Nature 499: 388.

8. Stevenson GW, Hoang H, Schwartz KJ, Burrough ER, Sun D, et al. (2013) Emergence of porcine epidemic diarrhea virus in the United States: Clinical signs, lesions, and viral genomic sequences. J Vet Diagn Investig 25: 649-654.

9. Chen Q, Li G, Stasko J, Thomas JT, Stensland WR, et al. (2014) Isolation and characterization of porcine epidemic diarrhea viruses associated with the 2013 disease outbreak among swine in the United States. J Clin Microbiol 52: 234-243.

10. Enjuanes L, Almazan F, Sola I, Zuniga S (2006) Biochemical aspects of coronavirus replication and virus-host interaction. Annu Rev Microbiol 60: 211-230.
11. Perlman S, Netland J (2009) Coronaviruses post-SARS: Update on replication and pathogenesis. Nat Rev Microbiol 7: $439-450$.

12. Graham RL, Baric RS (2010) Recombination, reservoirs, and the modular spike: Mechanisms of coronavirus cross-species transmission. J Virol 84: 3134-3146.

13. Li F (2013) Receptor recognition and cross-species infections of SARS coronavirus. Antivir Res 100: 246-254.

14. Li WH, Wong SK, Li F, Kuhn JH, Huang IC, et al. (2006) Animal origins of the severe acute respiratory syndrome coronavirus: Insight from ACE2-S-protein interactions. J Virol 80: 4211-4219.

15. Kirchdoerfer RN, Cottrell CA, Wang N, Pallesen J, Yassine $\mathrm{HM}$, et al. (2016) Pre-fusion structure of a human coronavirus spike protein. Nature 531: 118-121.

16. Walls AC, Tortorici MA, Bosch BJ, Frenz B, Rottier PJ, et al. (2016) Cryo-electron microscopy structure of a coronavirus spike glycoprotein trimer. Nature 531: 114-117.

17. Beniac DR, Andonov A, Grudeski E, Booth TF (2006) Architecture of the SARS coronavirus prefusion spike. Nat Struct Mol Biol 13: 751-752.

18. Li F, Berardi M, Li WH, Farzan M, Dormitzer PR, et al. (2006) Conformational states of the severe acute respiratory syndrome coronavirus spike protein ectodomain. $\mathrm{J}$ Virol 80: 6794-800.

19. Grodzki AC, Berenstein E (2010) Antibody purification: Ammonium sulfate fractionation or gel filtration. In: C Oliver, MC Jamur, Immunocytochemical Methods and Protocols, Methods in Molecular Biology. Humana Press, 588: 15-26.

20. Harlow E, Lane D (1988) Antibodies: A laboratory manual. Cold Spring Harbor Laboratory Press, Cold Spring Harbor, New York.

21. Gagnon PS (1996) Purification tools for monoclonal antibodies. Validated Biosystems.

22. Tripp RA, Haynes LM, Moore D, Anderson B, Tamin A, et al. (2005) Monoclonal antibodies to SARS-associated coronavirus (SARS-CoV): Identification of neutralizing and antibodies reactive to $\mathrm{S}, \mathrm{N}, \mathrm{M}$ and $\mathrm{E}$ viral proteins. J Virol Methods 128: 21-28. 